\title{
AN ELEMENTARY PROOF OF VOICULESCU'S ASYMPTOTIC FREENESS FOR RANDOM UNITARY MATRICES
}

\author{
Don Hadwin, Weihua Li and Junhao Shen
}

\begin{abstract}
D. Voiculescu [2] proved that a standard family of independent random unitary $k \times k$ matrices and a constant $k \times k$ unitary matrix is asymptotically free as $k \rightarrow \infty$. This result was a key ingredient in Voiculescu's proof [3] that his free entropy is additive when the variables are free. In this paper, we give a very elementary proof of a more detailed version of this result [2]. We have not yet recaptured Voiculescu's strengthened version [4].
\end{abstract}

Mathematics subject classification (2000): 46L10.

Keywords and phrases: asymptotic freeness, Haar measure, unitary.

\section{REFERENCES}

[1] M. Dostál And D. Hadwin, An alternative to free entropy for free group factors, International Workshop on Operator Algebra and Operator Theory (Linfen, 2001), Acta Math.Sin. (Engl. Ser.), 193 (2003), 419-472.

[2] D. Voiculescu, Limit laws for random matrices and free products, Invent. Math., 104 (1991), 201220.

[3] D. Voiculescu, The analogues of entropy and of fisher's information measure in free probability theory II, Invent. Math., 118 (1994), 411-440.

[4] D. VOICULESCU, Strengthened asymptotic freeness result for random matrices with applications to free entropy, Internat. Math. Res. Notices, 1 (1998), 41-63. 